ARTICLE

Received 2 Sep 2014 | Accepted 11 Feb 2015 | Published 20 Mar 2015

DOI: $10.1038 /$ ncomms7601

\title{
Genome-wide identification of microRNA expression quantitative trait loci
}

Tianxiao Huan ${ }^{1,2}$, Jian Rong ${ }^{3}$, Chunyu Liu' ${ }^{1,2}$, Xiaoling Zhang ${ }^{1,4}$, Kahraman Tanriverdi ${ }^{5}$, Roby Joehanes ${ }^{1,2,6,7,8}$, Brian H. Chen ${ }^{1,2}$, Joanne M. Murabito ${ }^{1,9}$, Chen Yao ${ }^{1,2}$, Paul Courchesne ${ }^{1,2}$, Peter J. Munson6, Christopher J. O'Donnell ${ }^{1,4}$, Nancy Cox ${ }^{10}$, Andrew D. Johnson ${ }^{1,4}$, Martin G. Larson ${ }^{1,3}$, Daniel Levy $y^{1,2}$ \& Jane E. Freedman ${ }^{5}$

Identification of microRNA expression quantitative trait loci (miR-eQTL) can yield insights into regulatory mechanisms of microRNA transcription, and can help elucidate the role of microRNA as mediators of complex traits. Here we present a miR-eQTL mapping study of whole blood from 5,239 individuals, and identify 5,269 cis-miR-eQTLs for 76 mature microRNAs. Forty-nine per cent of cis-miR-eQTLs are located 300-500 kb upstream of their associated intergenic microRNAs, suggesting that distal regulatory elements may affect the interindividual variability in microRNA expression levels. We find that cis-miR-eQTLs are highly enriched for cis-mRNA-eQTLs and regulatory single nucleotide polymorphisms. Among 243 cis-miR-eQTLs that were reported to be associated with complex traits in prior genomewide association studies, many cis-miR-eQTLs miRNAs display differential expression in relation to the corresponding trait (for example, rs7115089, miR-125b-5p and high-density lipoprotein cholesterol). Our study provides a roadmap for understanding the genetic basis of miRNA expression, and sheds light on miRNA involvement in a variety of complex traits.

\footnotetext{
${ }^{1}$ The Framingham Heart Study, Framingham, Massachusetts 01702, USA. ${ }^{2}$ The Population Sciences Branch, Division of Intramural Research, National Heart, Lung, and Blood Institute, Bethesda, Maryland 20824, USA. ${ }^{3}$ Department of Mathematics and Statistics, Boston University, Boston, Massachusetts 02118, USA. ${ }^{4}$ Cardiovascular Epidemiology and Human Genomics Branch, Division of Intramural Research, National Heart, Lung and Blood Institute, Bethesda, Maryland 20824, USA. ${ }^{5}$ Department of Medicine, University of Massachusetts Medical School, Worcester, Massachusetts 01655, USA. ${ }^{6}$ Mathematical and Statistical Computing Laboratory, Center for Information Technology, National Institutes of Health, Bethesda, Maryland 20824, USA. ${ }^{7}$ Harvard Medical School, Harvard University, Boston, Massachusetts 02115, USA. ${ }^{8}$ Hebrew SeniorLife, Boston, Massachusetts 02131, USA. ${ }^{9}$ Department of Medicine, Section of General Internal Medicine, Boston University School of Medicine, Boston, Massachusetts 02118, USA. ${ }^{10}$ Department of Human Genetics, University of Chicago, Chicago, Illinois 60637, USA. Correspondence and requests for materials should be addressed to D.L. (email: Levyd@nih.gov) or to J.E.F. (email: jane.freedman@umassmed.edu).
} 
$\mathrm{M}$ icroRNAs (miRNAs), a class of small noncoding RNAs, serve as key post-transcriptional regulators of gene expression and mRNA translation ${ }^{1,2}$. miRNAs are increasingly recognized as mediators in a variety of biological processes including cardiovascular development and disorders ${ }^{3,4}$. Highly specific miRNA expression patterns have been reported in association with heart failure ${ }^{5,6}$, myocardial infarction ${ }^{7}$ and cancer ${ }^{8}$. However, the influence of genetic variation on miRNA expression and function still remains unclear.

Recently, many genome-wide expression quantitative trait locus (eQTL) mapping studies have revealed common genetic loci associated with mRNA expression levels of many genes ${ }^{9-12}$. These eQTL analyses have demonstrated that transcript levels of many mRNAs behave as heritable quantitative traits. In contrast to more extensive investigations of mRNA eQTLs in multiple tissues $^{13}$ such as blood ${ }^{9}$, brain $^{10}$, fat $^{11}$ and liver ${ }^{12}$, there are relatively few studies of miRNA eQTLs (miR-eQTLs) and those that have been published to date are based on modest sample sizes $(n<200)^{14-18}$. These studies have identified relatively few cis-miR-eQTLs; uncertainty persists regarding the number of miR-eQTLs in humans and their relations to regulatory elements in the human genome.

We conduct a genome-wide miR-eQTL study by utilizing genome-wide genotypes and miRNA expression profiling of whole blood derived RNA from 5,239 Framingham Heart Study (FHS) participants. We analyze the associations of $\sim 10$ million 1000 Genomes Project ${ }^{19}$ imputed single nucleotide polymorphisms (SNPs) (at minor allele frequency (MAF) $>0.01$ and imputation quality ratio $>0.1$ ) with whole blood-derived miRNA expression levels of 280 mature miRNAs expressed in $>200$ individuals, representing $11 \%$ of all discovered human miRNAs to date (2,576 mature miRNAs have been reported in miRbase v20: www.mirbase.org). We calculate both cis- and trans-miR-eQTLs genome wide, and identify cis-miR-eQTLs with concordant effects in two pedigree independent study groups. By cross-linking cismiR-eQTLs SNPs with regulatory SNPs annotated by the ENCODE project $^{20}$ and with complex trait-associated SNPs identified in prior genome-wide association studies (GWAS) ${ }^{21,22}$, and by linking cis-miR-eQTL miRNAs with differentially expressed miRNAs for complex traits, we sought to dissect the genetic regulation of miRNA expression and explore the extent to which cis-miR-eQTLs may affect interindividual phenotype variability.

\section{Results}

Heritability of global miRNA expression in peripheral blood. The demographic and clinical characteristics of the 5,239 FHS participants included in our analysis are shown in Supplementary Data 1 . The pedigree structure formed by these participants is shown in Supplementary Data 2. We detected 280 mature miRNAs that were expressed in $>200$ participants (these miRNAs were used for identification of miR-eQTLs and unless specifically stated, miRNAs mentioned in the results and discussion refers to mature miRNAs), of these 247 miRNAs were expressed in $>1,000$ participants (Supplementary Fig. 1). The distribution of narrow-sense heritability of miRNA expression for the 247 miRNAs expressed in $>1,000$ participants is shown in Supplementary Fig. 2, with an average heritability estimate $\left(h_{\mathrm{miR}}^{2}\right)$ of $0.11 ; 133$ miRNAs $(54 \%)$ had $h_{\mathrm{miR}}^{2}>0.1$ and 9 miRNAs (miR100-5p, miR-668, miR-133a, miR-127-3p, miR-409-3p, miR-20a$3 p$, miR-941, miR-191-3p and miR-1303) had $h_{\mathrm{miR}}^{2}>0.3$ (details in Supplementary Data 3).

Cell type effects and reproducibility of miR-eQTLs. To evaluate whether blood cell type proportions significantly influence miR-eQTLs, we compared miR-eQTLs identified in 2,138 FHS third generation cohort participants (in whom differential cell counts and proportion data were available) with and without adjustment for measured blood cell counts and cell type proportions (see Methods). Cell types did not appreciably influence miR-eQTLs (Supplementary Fig. 3), however, we cannot exclude the possibility of small cell type effects. In the subsequent sections, we focus on miR-eQTLs from analyses that were unadjusted for cell counts of 5,239 individuals (Supplementary Data 4). The miR-eQTLs from the model that adjusted for imputed cell counts in the larger set of 5,024 participants are provided in Supplementary Data 5.

To evaluate the reproducibility of detected miR-eQTLs, we split our overall sample set 1:1 into two sets by pedigrees creating separate discovery and replication sets, and identified cis- and trans-miR-eQTLs in each set. At discovery false discovery rates (FDRs) of $<0.1,<0.05$ and $<0.01$, the replication rates for cismiR-eQTLs were $53 \%, 56 \%$ and $68 \%$ respectively, at a replication $\mathrm{FDR}<0.1$, and $100 \%$ showed allele-specific directional effect concordance between the discovery and replication sets (Fig. 1a,b). In contrast, no trans-miR-eQTLs replicated (at FDR $<0.1$ ), although $91 \%$ of trans-miR-eQTLs showed allelespecific directional effect concordance in the discovery and replication sets (Supplementary Fig. 4). Therefore, in the subsequent sections, we mainly report cis-miR-eQTLs identified in the overall FHS set (unadjusted for cell counts).

Genome-wide identification of miR-eQTLs. At $\mathrm{FDR}<0.1$ (corresponding $P$ value threshold is $6.6 \times 10^{-5}$ ), we identified
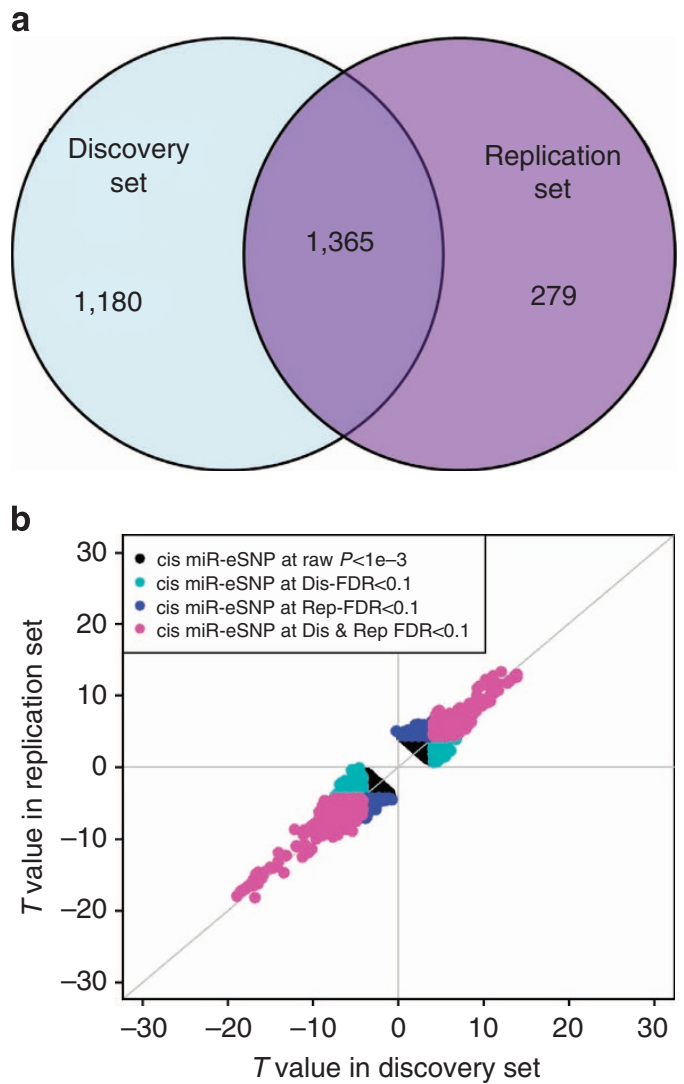

Figure 1 | Genome-wide identification of cis-miR-eQTLs. (a) Venn diagram of cis-miR-eQTLs identified in pedigree independent discovery $(n=2,671)$ and replication sets $(n=2,658)$. The number indicated cis-miReQTLs identified in discovery, replication or both at FDR $<0.1$. (b) $T$ values of cis-miR-eQTLs between discovery and replication groups. 
5,269 cis-miR-eQTLs for 76 miRNAs $(27 \%$ of interrogated expressed miRNAs) (Fig. 2). These cis-miR-eQTLs were further pruned by removing redundant cis-miR-eQTLs with high linkage disequilibrium (LD). At a series of LD $r^{2}$ thresholds, that is, $r^{2}=0.2,0.5,0.7,0.9$ and 1 , there were $283,572,982,1,602$ and 2,727 cis-miR-eQTLs retained. We further narrowed down the list to 52 peak cis-miR-eQTLs representing the single top cis-miReQTL for each miRNA or miRNA cluster shown in Supplementary Data 6. Table 1 shows 16 of the 52 peak cis-miReQTLs with GWAS SNPs in the NHGRI GWAS Catalogue and the NHLBI GRASP data set ${ }^{21,22}$. A miRNA cluster is defined as a group of miRNAs located within $10 \mathrm{~kb}$ in the same chromosome (using the criteria described in www.mirbase.org). miRNAs with higher heritability estimates were more likely to have cis-miReQTLs. All of the nine miRNAs with $h_{m i R}^{2}>0.3$ were found to have cis-miR-eQTLs. The top cis-miR-eQTLs tended to explain a greater proportion of the variance in the respective miRNA transcript as a function of increasing $h_{\mathrm{miR}}^{2}$ (Fig. 3). When the heritability of miRNA transcripts $h_{\mathrm{miR}}^{2}$ increased from (0 to 0.1 ) to $(0.3$ to 1$)$, the proportion of variance of the miRNA transcript explained by single cis-miR-eQTLs $\left(h_{\mathrm{miReQTL}}^{2}\right)$ increased from 0.02 to 0.08 on average.

At $\mathrm{FDR}<0.10$ (corresponding $P$ value threshold is $\left.1.0 \times 10^{-8}\right)$, we identified 270 trans-miR-eQTLs for 15 miRNAs ( $5 \%$ of interrogated expressed miRNAs). Supplementary Figure 5 showed two-dimensional regional plot of cis- and tran-miReQTLs genome widely (unadjusted cell counts). Supplementary Data 7-8 showed trans-miR-eQTLs at FDR $<0.1$ identified in the overall samples of adjusted and unadjusted cell counts, respectively. We acknowledged those trans-miR-eQTLs that need to be validated in independent cohorts.

cis-miR-eQTLs showing $\mathbf{5}^{\prime}$ positional bias for miRNAs. Among the 76 mature miRNAs with cis-miR-eQTLs, 49 (64\%) were intragenic, located within annotated protein-coding genes (located in exons, introns or untranslated regions of the host genes) and $27(36 \%)$ were intergenic. We discovered a marked positional bias of cis-miR-eQTLs, with many cis-miR-eQTLs located in the $5^{\prime}$-upstream region of the corresponding miRNA rather than within miRNA coding regions or the $3^{\prime}$-downstream regions.

Among the 982 non-redundant (LD $\left.r^{2}<0.7\right)$ cis-miR-eQTLs (representing 1,984 SNP-miRNA pairs), the relative distance of cis-miR-eQTLs to the corresponding mature miRNAs is shown in Fig. 4 and the relative distance of cis-miR-eQTLs to the transcriptional start site (TSS) is shown in Supplementary Fig. 6. Specifically, for intragenic miRNAs, 418 cis-miR-eQTLs (493 SNP-miRNA pairs, 58\%) were located in the $5^{\prime}$-upstream region of the corresponding primary miRNAs and 432 cis-miR-eQTLs (536 SNP-miRNA pairs, 63\%) were in the region defined by $200 \mathrm{~kb}$ upstream to $100 \mathrm{~kb}$ downstream of the TSS. In contrast, for intergenic miRNAs, 238 cis-miR-eQTLs (825 SNP-miRNA pairs, $83 \%$ ) were located in the $5^{\prime}$-upstream region of the corresponding primary miRNAs, and 125 cis-miR-eQTLs (487 SNP-miRNA pairs, 49\%) were in the region defined by 500 to $300 \mathrm{~kb}$ upstream of the TSS (Supplementary Data 9). There were 207 cis-miR-eQTLs (247 SNP-miRNA pairs, 29\%) for intragenic miRNAs and 99 cis-miR-eQTLs (129 SNP-miRNA pairs, 13\%) for intergenic miRNAs located within $\pm 50 \mathrm{~kb}$ of the TSS of the corresponding miRNAs.

Genomic features of cis-miR-eQTLs. Most of the detected cis-miR-eQTLs are not located in protein-coding regions, that is, $39 \%$ of eQTLs in intronic and $57 \%$ in intergenic regions (Supplementary Data 10). We found significant enrichment of cis-miR-eQTLs with expression regulatory elements (Table 2, Supplementary Data 11 and Supplementary Fig. 7), including CpG islands (2\%), promoters (9\%), enhancers (35\%) and transcription factor (TF) binding regions (15\%). We also found that cis-miR-eQTLs were enriched for miRNA mediated/targeted gene regulatory regions ${ }^{23,24}$.

There were $1,066(20 \%)$ cis-miR-eQTLs that overlapped with cis-mRNA-eQTLs identified in whole blood (enrichment $P<1 \mathrm{e}-300$ by hypergeometric test $)^{9,25}$. An example is shown in Supplementary Fig. 8; 132 cis-miR-eQTLs (36\%) for 12 intergenic mature miRNAs were also cis-mRNA-eQTLs for upstream protein-coding genes. We overlapped the $1 \mathrm{Mb}$ region flanking the 132 cis-miR-eQTLs (chr14: 100.5-102.5 Mb) with the regulatory feature tracks downloaded from UCSC Genome Browser (genome.ucsc.edu). Supplementary Figure 8 showed that the nearby regions of the 132 cis-miR-eQTLs for those 12 miRNAs overlap with Enhancer active region (chr14:101,100 kb$101,200 \mathrm{~kb}, \mathrm{H} 3 \mathrm{~K} 4 \mathrm{Me} 1$ and H2K27AC track, marked in lightyellow rectangle). The highly unmethylated status of GM12878, K562, HeLa-S3 and HepG2 cell lines are in chr14:101,400 kb-101,600 kb upstream of those cis-mRNAeQTL miRNAs (CpG Methylation by Methy450K Bead Arrays from ENCODE/HAIB track, marked by pink colour).

We also discovered 11 intragenic mature miRNAs share cis-eQTLs with their host mRNA genes (Supplementary Data 12).

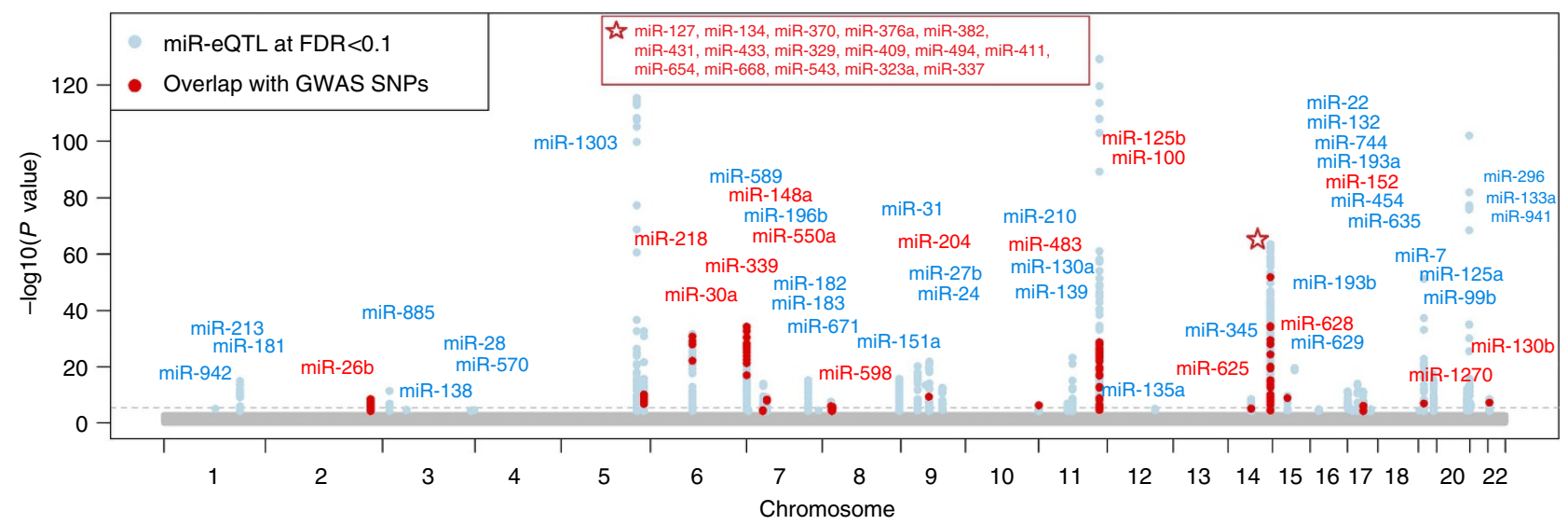

Figure 2 | Manhattan plot of cis-miR-eQTLs. Genome-wide - $\log 10(P)$ value plots are shown for every interrogated miRNA (280 expressed miRNAs). Seventy six miRNAs having cis-miR-eQTLs are labelled in this figure (for 52 unique peak loci). The horizontal dotted line indicates FDR $<0.1$ (corresponding to $P<6.6 \times 10^{-5}$ ). cis-miR-eQTL SNPs overlapping with GWAS SNPs reported in NHGRI GWAS Catalogue (http://www.genome.gov/gwastudies/) ${ }^{21}$ and NHLBI GRASP database (http://apps.nhlbi.nih.gov/grasp/) 22 are shown in red. 


\begin{tabular}{|c|c|c|c|c|c|c|c|c|}
\hline $\begin{array}{l}\text { Peak cis- } \\
\text { miR-eQTL }\end{array}$ & Chr. & miRNAs & $\begin{array}{l}\text { Genome } \\
\text { context } \\
\text { (miRNA) }\end{array}$ & $\begin{array}{l}\text { miR- } \\
\text { eQTL } \\
\text { FDR }\end{array}$ & $\begin{array}{l}\text { Proxy miR-eQTLs } \\
\text { overlap with } \\
\text { GWAS SNPs } \\
\end{array}$ & GWAS traits & $\begin{array}{l}\text { GWAS } \\
P \text { value }\end{array}$ & $\begin{array}{l}\text { Trait signature } \\
\text { miRNAs }\end{array}$ \\
\hline \multirow[t]{2}{*}{ rs7607369 } & chr2 & miR-26b-5p & $\begin{array}{l}\text { Intron } \\
\text { (CTDSP1) }\end{array}$ & $1.2 e-5$ & rs1541777 & Height & $8.6 e-9$ & - \\
\hline & & & & & rs2241527 & $\begin{array}{l}\text { Severe statin-induced } \\
\text { myopathy }\end{array}$ & $1.3 e-6$ & - \\
\hline rs9342836 & chr6 & miR-30a-3p & $\begin{array}{l}\text { Intron } \\
\text { (C6orf155) }\end{array}$ & $8.3 e-28$ & rs7349905 & Kawasaki disease & $5.4 e-6$ & - \\
\hline rs11763835 & chr7 & miR-339-3p, miR-339-5p & $\begin{array}{l}\text { Intron } \\
(C 70 r f 50)\end{array}$ & $2.5 e-30$ & rs6951245 & Total cholesterol & $6.1 e-8$ & $\begin{array}{c}\operatorname{miR}-339-3 p \\
(P=2.5 e-7)\end{array}$ \\
\hline rs1839612 & chr7 & miR-550a-3p & $\begin{array}{l}\text { Intron } \\
\text { (ZNRF2) }\end{array}$ & $2.3 e-6$ & $\begin{array}{l}\text { rs13242526 } \\
\text { rs2060708 }\end{array}$ & $\begin{array}{l}\text { LDL cholesterol } \\
\text { White blood cell } \\
\text { count (WBC) }\end{array}$ & $\begin{array}{l}7.7 e-6 \\
3.9 e-6\end{array}$ & $\begin{array}{l}- \\
-\end{array}$ \\
\hline rs17747335 & & & & & rs10090800 & $\begin{array}{l}\text { Second to fourth digit } \\
\text { length ratio }\end{array}$ & $5.3 e-6$ & - \\
\hline rs28640110 & chr9 & miR-204-5p & $\begin{array}{l}\text { Intron } \\
\text { (TRPM3) }\end{array}$ & $2.6 e-18$ & rs2993008 & $\begin{array}{l}\text { Common variable } \\
\text { immunodeficiency } \\
\text { (Splenectomy) }\end{array}$ & $2.5 e-6$ & - \\
\hline \multirow[t]{3}{*}{ rs2370747 } & chr11 & miR-100-5p, miR-125b-5p & Intergenic & $1.8 e-130$ & rs7115089 & Total cholesterol & $3.2 \mathrm{e}-10$ & $\operatorname{miR}-125 b-5 p$ \\
\hline & & & & & & HDL cholesterol & $8.4 e-9$ & $\begin{array}{c}\text { miR-100-5p } \\
(P=0.039) ; m i R-125 b \\
5 p(P=1.68 \mathrm{e}-5)\end{array}$ \\
\hline & & & & & rs7941030 & $\begin{array}{l}\text { LDL cholesterol } \\
\text { Multiple sclerosis }\end{array}$ & $\begin{array}{l}7.6 e-6 \\
8.2 e-6\end{array}$ & - \\
\hline rs11042699 & chr11 & miR-483-3p & $\begin{array}{l}\text { Intron } \\
(I G F 2)\end{array}$ & $9.6 e-4$ & rs6578985 & $\begin{array}{l}\text { Coronary artery disease } \\
\text { (CAD) }\end{array}$ & $1.6 e-6$ & - \\
\hline rs4905998 & chr14 & $\begin{array}{l}\text { miR-127-3p, miR-134, miR-370, } \\
\text { miR-376a-3p, miR-382-5p, } \\
\text { miR-431-5p, miR-433, } \\
\text { miR-329, miR-409-3p, } \\
\text { miR-494, miR-411-3p, } \\
\text { miR-654-5p, miR-668, } \\
\text { miR-543, miR-323a-3p, } \\
\text { miR-337-3p }\end{array}$ & Intergenic & $2.7 e-59$ & rs6575793 & Age at menarche & $1.7 e-10$ & $\begin{array}{c}\text { miR-376a-3p } \\
(P=0.007) ; \mathrm{miR}-382 \\
5 p(P=0.046)\end{array}$ \\
\hline rs2127868 & & & & & rs1256526 & Type 2 diabetes & $4.7 e-6$ & $\begin{array}{l}\operatorname{miR}-625-5 p \\
(P=0.035)\end{array}$ \\
\hline rs28483325 & chr15 & miR-628-3p & $\begin{array}{l}\text { Intron } \\
\text { (CCPG })\end{array}$ & $8.1 e-7$ & rs 7168869 & $\begin{array}{l}\text { Mean corpuscular volume } \\
(\mathrm{MCV})\end{array}$ & $4.3 e-6$ & - \\
\hline \multirow[t]{3}{*}{ rs2737 } & chr17 & miR-152 & $\begin{array}{l}\text { Intron } \\
(C O P Z 2)\end{array}$ & $3.1 e-8$ & rs1553754 & Body mass index (BMI) & $2.8 e-6$ & - \\
\hline & & & & & rs11079828 & $\begin{array}{l}\text { Attention-deficit/ } \\
\text { hyperactivity } \\
\text { disorder (ADHD) }\end{array}$ & $6.5 e-6$ & - \\
\hline & & & & & rs 6504340 & $\begin{array}{l}\text { Primary Tooth Development } \\
\text { during Infancy (Number of } \\
\text { teeth by } \\
\text { one year of age) }\end{array}$ & $6.1 e-7$ & - \\
\hline rs28576121 & chr19 & $\operatorname{miR}-1270$ & $\begin{array}{l}\text { Intron } \\
\text { (ZNF826P) }\end{array}$ & $2.1 e-50$ & rs7251204 & Fasting blood glucose & $4.0 e-6$ & $\operatorname{miR}-1270(P=0.002)$ \\
\hline rs373001 & chr22 & miR-130b-5p, miR-130b-3p & $\begin{array}{l}\text { Exon } \\
(\text { PPIL2) }\end{array}$ & $1.1 e-5$ & $\begin{array}{l}\text { rs2562664 } \\
\text { rs861844 }\end{array}$ & $\begin{array}{l}\text { Fasting insulin } \\
\text { Myocardial infarction (MI), } \\
\text { sudden cardiac arrest in } \\
\text { patients with } \\
\text { coronary artery disease } \\
\text { (CAD) }\end{array}$ & $\begin{array}{l}8.7 e-6 \\
5.3 e-6\end{array}$ & \\
\hline
\end{tabular}

For cis-miR-eQTLs that overlapped with cis-mRNA-eQTLs, we performed conditional analysis to test if the associations between SNPs and miRNAs remained significant when conditioning on the corresponding mRNA expression levels using results from 5024 FHS participants with genotype, and miRNA, and mRNA expression data. As show in Supplementary Data 13, we found 923 cis-miR-eQTLs for 3,384 miRNA-SNP association pairs $(87 \%)$ that remained significant at $\mathrm{FDR}<0.1$ (corresponding
$\left.P<6.6 \times 10^{-5}\right)$ when conditioning on mRNA expression levels. These findings indicate that cis genetic variants may affect expression levels of neighbouring miRNAs and mRNAs.

cis-miR-eQTLs and miRNA signatures for complex traits. We linked the cis-miR-eQTLs with GWAS SNPs in the NHGRI GWAS Catalogue and the NHLBI GRASP data set $^{21,22}$. Among 


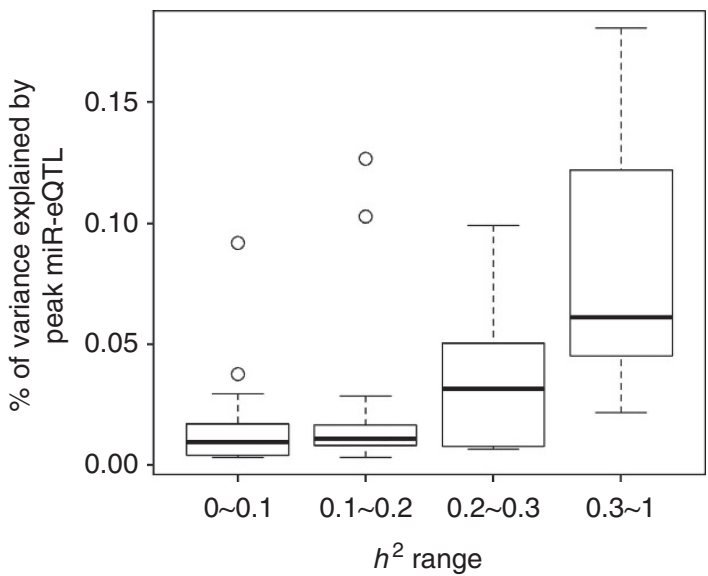

Figure 3 | The variance proportion of miRNA expression explained by single cis-miR-eQTLs at different heritability levels. This figure was plotted by the boxplot function in the R library. The boxes indicate the interquartile range (IQR) of data between $75 \%(Q 3)$ and $25 \%$ (Q1). The bars below and above each box indicate the data in Q1 $1.5 \times \mathrm{IQR}$ and $\mathrm{Q} 3+1.5 \times \mathrm{IQR}$, respectively.

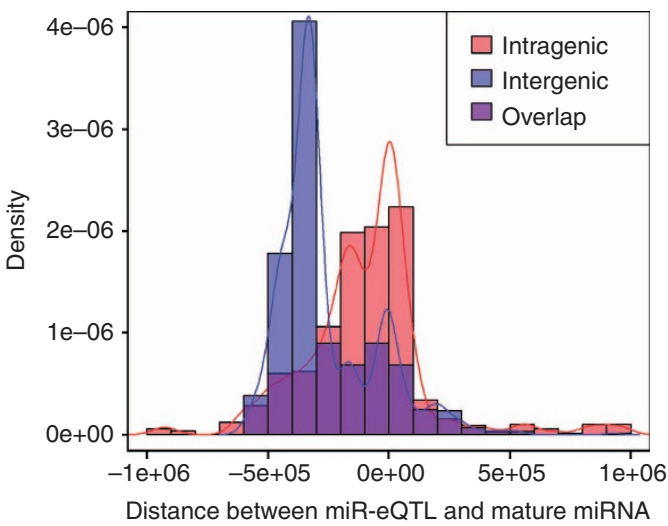

Figure 4 | The distribution of distance between cis-miR-eQTLs and miRNA position. cis-miR-eQTLs for intergenic miRNAs are generally located further upstream than for intragenic miRNAs. The position of the first nuclear acid of the mature miRNA is marked as 0 . The distribution statistics are based on 982 unique cis-miR-eQTLs with LD $r^{2}<0.7$.

5,269 cis-miR-eQTLs, 243 cis-miR-eQTLs (for 31 miRNAs) overlapped with GWAS SNPs, including SNPs associated with multiple complex traits (Table 1, Fig. 2, and regional association plots for several traits including height, menarche, platelet count and lipid levels are shown in Supplementary Fig. 9).

For miRNAs with cis-miR-eQTLs showing association with complex traits in GWAS, we further tested if expression of these miRNAs in FHS participants was associated with the corresponding traits. We discovered a number of miRNAs that showed differential expression in relation to the complex traits that correspond to the traits associated with their eQTLs in GWAS (Table 1). For example (Fig. 5a,b), among cis-miR-eQTLs of miR$100-5 \mathrm{p}$ and miR-125b-5p, we found 28 cis-miR-eQTLs (that is, GWAS SNPs) that were associated in GWAS with lipid traits (high-density lipoprotein (HDL) cholesterol, low-density lipoprotein (LDL) cholesterol, total cholesterol (TC) and triglycerides (TGs)), 1 (rs7941030) with multiple sclerosis and 1 (rs1216554) with rheumatoid arthritis. These eQTLs are located $\sim 519 \mathrm{~kb}$ upstream of their two associated miRNAs. We also found that miR-125b-5p showed differential expression in relation to plasma

\begin{tabular}{|c|c|c|c|}
\hline Genome regulatory track & $\begin{array}{l}\text { Nucleotides } \\
\text { per track }\end{array}$ & $\begin{array}{c}\text { Fold } \\
\text { change }\end{array}$ & $P$ value ${ }^{\star}$ \\
\hline $\begin{array}{l}\text { UCSC CpG Islands } \\
\text { lincRNAs }\end{array}$ & $\begin{array}{l}21575631 \\
127119148\end{array}$ & $\begin{array}{l}2.6 \\
0.8\end{array}$ & $\begin{array}{c}2.97 e-16 \\
1\end{array}$ \\
\hline $\begin{array}{l}\text { Known regulatory elements } \\
\text { (Oreganno) }\end{array}$ & 11265267 & 3.2 & $7.48 e-15$ \\
\hline miRNA targets (TARbase) & 49662027 & 6.9 & $5.15 e-289$ \\
\hline $\begin{array}{l}\text { miRNA-mediated gene regulatory } \\
\text { sites (Patrocles) }\end{array}$ & 3375454 & 10.0 & $1.64 e-37$ \\
\hline GM12878 CTCF & 44516245 & 2.0 & $7.42 e-15$ \\
\hline GM128 & 125879 & 1.9 & $4.11 e-35$ \\
\hline $\mathrm{GM}$ & 1136357520 & 1.4 & $1.17 e-92$ \\
\hline GM12878 H3k36me3 & 631024019 & 1.6 & $2.80 e-106$ \\
\hline GM12 & 242340600 & 1.9 & $6.59 e-63$ \\
\hline GM12878 & 120458965 & 2.0 & $2.90 e-37$ \\
\hline
\end{tabular}

CTCF, CCCTC-binding factor; ChIP, chromatin immunoprecipitation; eQTL, expression quantitative trait loci; me, methylation; miR, microRNA; UCSC, University of California, Santa quantitative trait loci; me, methylation; miR, microRNA; UCSC, University of California, San
Cruz.

${ }^{\star} P$ values are for binomial tests for enrichment of observed over expected; GM12878 is a lymphoblastoid cell line; CTCF marks CTCF Binding Sites by ChIP-seq from ENCODE; H3k27ac and $\mathrm{H} 3 \mathrm{~K} 4 \mathrm{me} 1$ mark active/poised enhancers; $\mathrm{H} 3 \mathrm{~K} 4 \mathrm{me} 3$ marks active/poised promoters; and H3K36me3 marks actively transcribed regions.

TC $(P=0.005$, by linear regression tests, see Methods $)$ and HDL cholesterol $(P=1.68 \mathrm{e}-5)$ and miR-100-5p showed differential expression in relation to HDL cholesterol $(P=0.039)$. Another example (Fig. $5 c, d$ ) is for miR-339-3p and miR-339-5p, which are located in an intron of c7orf50. Among the 282 cis-miR-eQTLs SNPs of miR-339-3p and 279 cis-miR-eQTLs of miR-339-5p, 8 were associated with TC and 3 with LDL cholesterol. We also found that expression of miR-339-3p was associated with TC $(P=2.5 \mathrm{e}-7)$. These results establish links between SNPs affecting both miRNA expression levels and complex traits. Mendelian randomization (MR) tests provided evidence that four cis-miR-eQTLs SNPs (rs6951245, rs11763020, rs1997243 and rs2363286) alter the expression levels of miR-339-3p and miR339-5p, and in turn affect interindividual variability of TC levels (causal $P<0.05$ ).

\section{Discussion}

On the basis of extensive integrated analyses of miRNA expression and genetic variants genome wide in 5,239 individuals, we established a clear pattern of heritability of blood miRNA expression, and identified a substantial number of miRNAs that are controlled by cis genetic regulatory elements. Our results for cis-miR-eQTLs were highly replicable; in contrast, trans-miReQTLs were not replicable. Previously reported miR-eQTLs were identified in studies with small sample sizes $(n<200)$ and revealed a few miR-eQTLs. For example, Borel et al. ${ }^{14}$ using umbilical cord blood from 180 newborns, identified only 12 cismiR-eQTLs at $\mathrm{FDR}<0.5$. In another study, no cis-miR-eQTLs were found in 176 lymphoblastoid cell lines from European and African ancestry samples ${ }^{15}$. Proxy SNPs of two cis-miR-eQTLs that we identified (rs2187519 for miR-100 and rs7797405 for miR-550) were reported by Borel et al. ${ }^{14}$ ( $\mathrm{rs} 10750218$ as a proxy for rs2187519 and rs12670233 for rs7797405 are in modest LD at $r^{2}=0.29$ and $r^{2}=0.48$, respectively).

As our data are from a well-powered multi-generation study, we were able to assess narrow sense heritability $\left(h_{\mathrm{miR}}^{2}\right)$ of each miRNA expression trait. By comparing the overall heritability of the miRNAs and single cis-miR-eQTLs, we discovered that miRNAs with higher heritability were more likely to have cismiR-eQTLs. When the heritability of miRNA transcripts $h_{\mathrm{miR}}^{2}$ increased, the proportion of variance of the miRNA transcript 

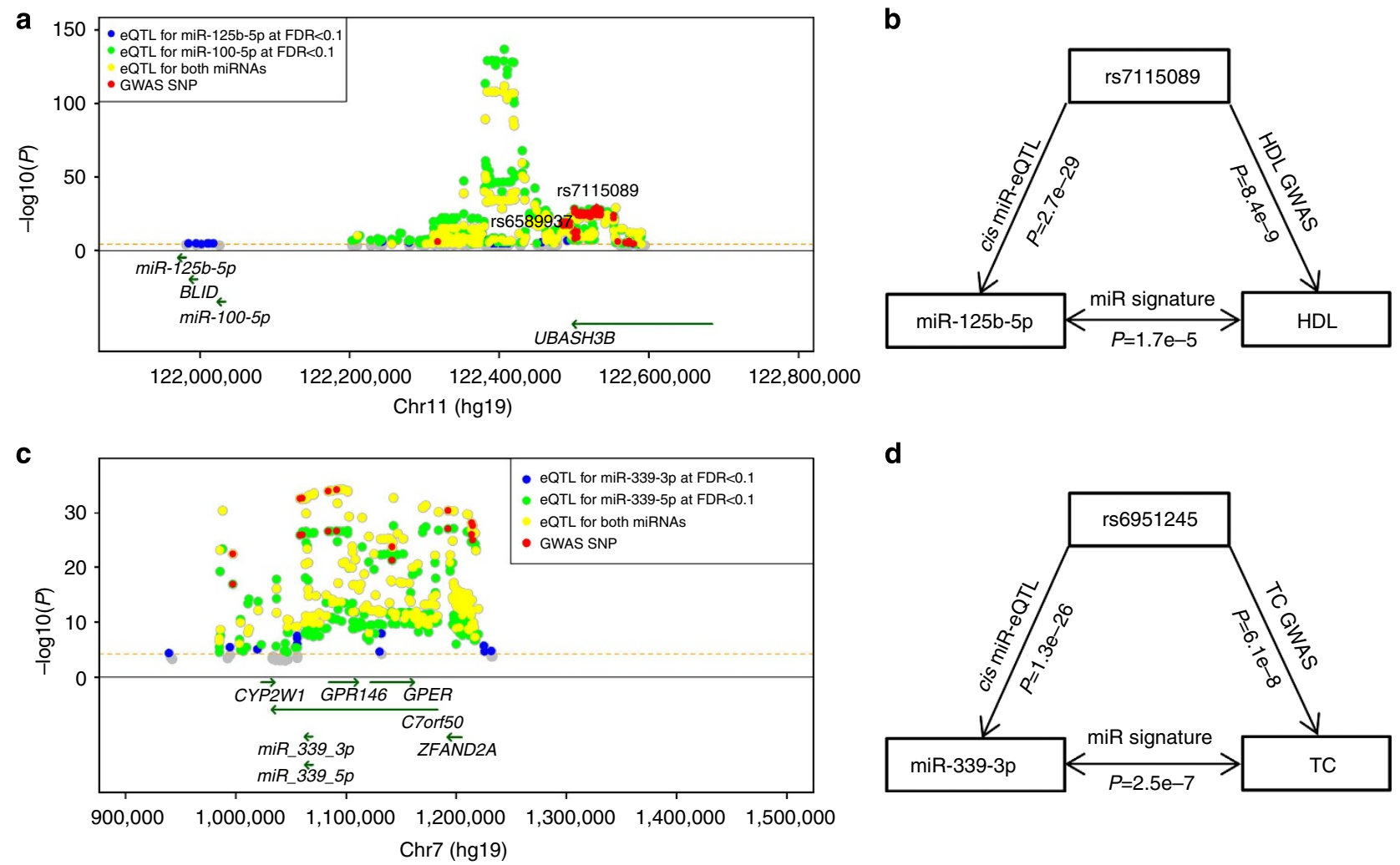

Figure 5 | Regional association plot of cis-miR-eQTLs that were associated with GWAS SNPs. (a) miR-eQTLs for intergenic miRNAs miR-100-5p and miR-125b-5p, with GWAS SNPs for lipid traits, multiple sclerosis and rheumatoid arthritis. The highlighted SNP, rs7115089, is associated with both HDL and total cholesterol at GWAS $P<5 \times 10^{-8}$ by linear regression tests ${ }^{50}$; (b) the triangular relationships between SNP (that is, rs7115089), miRNA (that is, miR-125b-5p) and HDL cholesterol; (c) miR-eQTLs for intragenic miRNAs miR-339-3p and miR-339-5p, with GWAS SNPs for TC and LDL; (d) the triangular relationships between SNP (that is, rs6951245), miRNA (that is, miR-339-3p) and TC. - $\log 10(P)$ indicates the -log10 transformed miRNA-SNP association $P$ values.

explained by single cis-miR-eQTLs $\left(h_{\mathrm{miR}-\mathrm{eQTL}}^{2}\right)$ increased as well. Our heritability study of mRNA expression traits revealed single cis-mRNA-eQTLs that explained $33-53 \%$ of variances in corresponding mRNA expression levels ${ }^{26}$. In contrast, single cis-miR-eQTLs explained much less proportion of variances in corresponding miRNA expression levels ( $\sim 1.3 \%$ on average).

In contrast to the functional annotation of cis-mRNA-eQTLs, most of which are within $\sim 250 \mathrm{~kb}$ of mRNA TSS and without $5^{\prime}$ or $3^{\prime}$ positional bias ${ }^{13}$, we discovered that most cis-miR-eQTLs (58\% for intragenic miRNAs and $83 \%$ for intergenic miRNAs) are located upstream of mature/primary miRNAs. For intergenic miRNAs, a significant fraction of cis-miR-eQTLs are quite far upstream $(\sim 300-500 \mathrm{~kb})$. Distal regulatory elements can interact with the proximal elements that regulate miRNA expression ${ }^{27}$. In our results, we found that a significant fraction of cis-miR-eQTLs are distal, suggesting that variants in far upstream regions may play important roles in miRNA transcription. In addition, our results revealed that distal cis-miR-eQTLs explained a modest proportion ( $\sim 1.3 \%$ on average) of variance in miRNA expression levels. We speculate that the mild effects of cis-miR-eQTLs on miRNA expression result from evolutionary selection to stabilize the biological functions mediated by miRNAs.

Genetic variants that modify chromatin accessibility and transcription factor binding are a major mechanism through which genetic variation leads to expression differences for proteincoding genes in humans ${ }^{28}$. The investigation of regulatory mechanisms of miRNA transcription is still evolving. Genomic feature analyses of cis-miR-eQTLs reveal that a large proportion of cis-miR-eQTLs are located in regulatory elements such as CpG islands (2\%), promoters (9\%), enhancers (35\%) and TF binding regions $(15 \%)$. We also discovered that cis-miR-eQTLs show a significant enrichment for mRNA-eQTLs and $87 \%$ of cis-miReQTLs that are also mRNA-eQTLs remained significant when conditioning on the corresponding mRNA expression levels. For example, as shown in Supplementary Fig. 8, 132 cis-miR-eQTLs (36\%) for 12 intergenic miRNAs were also cis-mRNA-eQTLs for upstream protein-coding genes. This finding suggests that genetic variants may influence the expression of both miRNAs and nearby protein-coding genes. These eQTL regulatory effects may act via modified chromatin accessibility, transcription factor binding affinity or DNA methylation.

The mechanisms of transcriptional regulation of intragenic miRNAs are more complex than intergenic miRNAs, as intragenic miRNAs may mirror the regulatory mechanisms of their host genes, or be transcribed independently as a consequence of their unique promoter regions ${ }^{29}$. We identified 11 mature miRNAs from intragenic miRNAs that share cis eQTLs with their host protein coding genes (Supplementary Data 12). Among the cis-mRNA-eQTL miRNAs, 15 miRNAs have alternative intronic promoters (alternative intronic promoters were from ref. 29). We overlapped the cis-miR-eQTLs and expression regulatory elements annotations from ENCODE nearby regions of each miRNA $( \pm 50 \mathrm{~kb})$. We found, in some examples (Supplementary Fig. 10), cis-miR-eQTLs near alternative intronic promoter regions demonstrated promoter and enhancer activities and were highly un-methylated in some cell lines. Our findings provide a guide for further functional studies of transcriptional elements of miRNAs. 
We identified numerous cis-miR-eQTLs that are associated with complex diseases/traits in GWAS (Table 1). Equally noteworthy, we found several examples in which the miRNAs associated in cis with these GWAS SNPs were associated with the corresponding trait (for example, three-way association of HDL cholesterol with its GWAS SNP, rs7115089 and with the corresponding miR-125b$5 p)$. A single miRNA may target hundreds of protein-coding genes. Therefore, the effect of genetic variants on miRNAs can play an important regulatory role in mediating the targeted protein-coding genes, as well as complex phenotypes. We speculate that some of the protein-coding genes targeted by miRNAs may also be involved in the cellular pathways related to the trait. For example, miR-125b-5p expression was associated with HDL cholesterol $\left(P=1.7 \times 10^{-5}\right.$, by a linear regression test). In a parallel project focusing on differentially expressed mRNAs in association with lipid levels, we found 17 genes targeted by miR125b-5p (9\% of miR-125b-5p targeted genes in miRTarBase ${ }^{24}$ ) that showed differential expression in association with HDL cholesterol (at $P<0.05$ corrected for $\sim 18,000$ genes, by a linear regression test $)^{30}$. Some of these genes are involved in metabolic processes, for example, $P R D X 2$, which was downregulated in association with $\mathrm{HDL}$ cholesterol $\left(P=1.1 \times 10^{-15}\right.$, by a linear regression test). Further studies and biological experiments are needed to investigate whether these cis-miR-eSNPs affect the corresponding miRNA targeting genes.

In summary, our genome-wide miR-eQTL mapping study provides new insights into the genetic regulation of miRNA transcription and the roles of miRNAs in complex diseases. Our findings may help to identify new opportunities for drug treatment or diagnosis of human diseases.

\section{Methods}

Study populations. The FHS is a community-based study that began enroling participants in 1948. In 1971, the offspring and offspring spouses (the offspring cohort) of original FHS cohort participants were recruited and they have been examined every 4 to 8 years ${ }^{31}$. From 2002 to 2005, the adult children of the offspring cohort participants (the third generation cohort) were recruited and examined $^{32}$. In this study, we investigated 2,272 offspring cohort attendees at examination cycle 8 (2005-2008) and 3,057 third generation cohort attendees at examination cycle 2 (2008-2010). This study was approved under the Boston University Medical Center protocol H-27984. Written informed consent was obtained from each participant.

miRNA expression profiling. miRNAs were measured from venous blood samples obtained from participants after overnight fasting. Whole blood samples $(2.5 \mathrm{ml})$ were collected in PAXgene Blood RNA tubes (Qiagen, Valencia, CA, USA) and frozen at $-800{ }^{\circ} \mathrm{C}$. Total RNA was isolated from the frozen PAXgene Blood RNA tubes (Asuragen, Inc. Austin, TX, USA) and a 2100 Bioanalyzer Instrument (Agilent, Santa Clara, CA, USA) was used for RNA quality assessment. Isolated RNA samples were converted to complementary DNA using TaqMan miRNA Reverse Transcription Kit and MegaPlex Human RT Primer Pool Av2.1 and Pool Bv3.0. (Life Technologies, Foster City, CA, USA) in a 384-well Thermal Cycler. The complementary DNA samples were PreAmplified using TaqMan PreAmp Master Mix and PreAmp Primers, Human Pool A v2.1 and Pool B v3.0 (Life Technologies)

Quantitative reverse transcription PCR ( $q R T-P C R$ ) reactions were performed with the BioMark System using (Fluidigm, South San Francisco, CA, USA) TaqMan miRNA Assays (Life Technologies). As described in the published literature, measurement of RNA by qRT-PCR is reliable and has high specificity and sensitivity ${ }^{33-36}$. The initial miRNA list encompassed all commercially available TaqMan miRNA assays obtainable at the start of the project (754 mature miRNAs). These miRNAs were initially assayed for measurement feasibility in RNA samples from 450 FHS participants. All qRT-PCR reactions were performed in the BioMark Real-Time PCR system using the following protocol: $10 \mathrm{~min}$ at $95^{\circ} \mathrm{C}, 15 \mathrm{~s}$ at $95^{\circ} \mathrm{C}$ and $1 \mathrm{~min}$ at $60^{\circ} \mathrm{C}$ for 30 cycles. Single copy can be detected with BioMark system at 26-27 cycle thresholds. For replicates $>95 \%$ of the data points had coefficients of variation $<10 \%$ (mean $\sim 4 \%$ ).

miRNA normalization. We normalized miRNA expression using a model that adjusts raw miRNA cycle threshold $(\mathrm{Ct})$ values for 4 technical variables: isolation batch (50 batches), RNA concentration, RNA quality (defined as RNA integrity number) and RNA 260/280 ratio (ratio of absorbance at 260 and $280 \mathrm{~nm}$; measured using a spectrophotometer). Histograms (Supplementary Fig. 11) show that this model explains $20-60 \%$ of variability of raw miRNA measurements for $80 \%$ of miRNAs

Genotyping. DNA was isolated from buffy coat or from immortalized lymphoblast cell lines. Genotyping was conducted with the Affymetrix $500 \mathrm{~K}$ mapping array and the Affymetrix $50 \mathrm{~K}$ gene-focused MIP array, using previously described quality control procedures ${ }^{37}$. Genotypes were imputed to the 1000 Genomes Project panel 19 of $\sim 36.3$ million variants using $\mathrm{MACH}^{38}$. We filtered out SNPs with $\mathrm{MAF}<0.01$ and imputation quality ratio $<0.1$ (the imputation quality ratio is denoted by the ratio of the variances of the observed and the estimated allele counts), resulting in $9.8 \times 10^{6}$ SNPs $(\sim 10$ million SNPs) that were eligible for further miR-eQTL testing.

miR-eQTL mapping. Because of the computational burden of running linear mixed effects (LMEs) models for $\sim 10$ million (SNPs) $\times 280$ miRNAs (miRNAs expressed in $>200$ samples), we adapted a two-step analysis strategy. Step 1: linear regression was used to model the association between miRNA Ct values (miR) and the imputed SNP genotypes-adjusted for age, sex, cohort and technical covariates-yielding results for roughly 280 miRNAs $\times 10$ million SNPs, as shown in Equation (1). Associated SNP-miRNA pairs residing within $1 \mathrm{Mb}$ of the mature miRNA (cis) and those residing more than $1 \mathrm{Mb}$ away (trans) were identified separately. We chose liberal $P$ value thresholds to pre-filter the miR-eQTLs, at $P<1 \times 10^{-3}$ for cis and $P<1 \times 10^{-5}$ for trans. These $P$ value thresholds were chosen to ensure that miR-eQTLs at a FDR $<0.1$ were not omitted as a result of this pre-filtering step. Step 2: we used a linear mixed model ${ }^{39}$ to recalculate the associations of SNPs and miRNA expression levels for the pre-selected eQTLs from step 1, adjusted for age, sex and technical covariates as fixed effects and a familial correlation matrix (FAM) as the random effect using the lmekin() function of Kinship Package (http://cran.r-project.org/web/packages/kinship/) ${ }^{39}$, as shown in Equation (2). In Equations (1) and (2), $\varepsilon$ is the error term for each independent observation.

$$
\operatorname{miR}=\mathrm{SNP}+\text { age }+ \text { sex }+ \text { cohort }+\sum_{i=1}^{n} \text { techical covariates }+\varepsilon
$$

$$
\mathrm{miR}=\mathrm{SNP}+\text { age }+\mathrm{sex}+\sum_{i-1}^{n} \text { techical covariates }+\mathrm{FAM}+\varepsilon
$$

Genome coordinate annotation for miRNAs used miRbase v20 (mirbase.org), and for SNPs we used the February 2009 assembly of the human genome (hg19, GRCh37 Genome Reference Consortium Human ${ }^{37}$ ). Based on the coordinates of 280 mature miRNAs and $9.8 \times 10^{6} \mathrm{SNPs}$, we estimated there were $13,935,272$ $\left(1.4 \times 10^{7}\right)$ potential SNP-miRNA pairs, where the SNP was located within $1 \mathrm{Mb}$ on either side of the corresponding mature miRNA. We estimated there were $1.4 \times 10^{7}$ potential cis SNP-miRNA pairs, and $2.7 \times 10^{9}$ (that is, $280 \times 9.8 \times 10^{6}-1.4 \times 10^{7}$ ) potential trans SNP-miRNA pairs. We used the Benjamini-Hochberg method ${ }^{40}$ to calculate FDR for cis- and trans-miR-eQTLs by correcting for $1.4 \times 10^{7}$ potential cis $\mathrm{SNP}$-miRNA pairs and $2.7 \times 10^{9}$ potential trans SNP-miRNA pairs, respectively. We selected an FDR threshold of 0.1, corresponding to $P<6.6 \times 10^{-5}$ for cis- and $P<1.0 \times 10^{-8}$ for trans-miR-eQTLs.

For identified cis-miR-eQTLs at FDR $<0.1$, we used Fragmented Exhaustive Search for TAgSNPs (FESTA) ${ }^{41}$ to select non-redundant miR-eQTLs based on a series of LD $r^{2}$ thresholds $0.2,0.5,0.70 .9$ and 1. FESTA used a mixture of search techniques to partition the whole SNP set into disjointed precincts and selected a tag SNP for each SNP block, which represented a set of SNPs at a LD $r^{2}$ $>$ threshold $^{41}$.

To estimate the replicability of miR-eQTLs, we split the overall sample set at a 1:1 ratio into discovery and replication sets. The discovery and replication sets represent independent pedigrees to ensure that individuals in the two sets were unrelated. We used the methods described above to identify miR-eQTLs in the discovery and replication sets separately. We evaluated the concordance of effect sizes of cis- and trans-miR-eQTLs in the discovery and replication sets. We identified eQTLs at FDR $<0.1$ in the discovery set, and attempted to replicate them in the replication set.

mRNA expression data. Whole blood samples $(2.5 \mathrm{ml})$ were collected in PAXgene tubes by Asuragen, Inc. (PreAnalytiX, Hombrechtikon, Switzerland). Total RNA was isolated according to the company's standard operating procedures for automated isolation of RNA from 96 samples in a single batch on a KingFisher 96 robot. Then 50 ng RNA samples were amplified using the WT-Ovation Pico RNA Amplification System (NuGEN, San Carlos, CA, USA) as recommended by the manufacturer in an automated manner using the genechip array station. RNA expression was conducted using the Affymetrix Human Exon Array ST 1.0 (Affymetrix, Inc., Santa Clara, CA, USA). The core probe sets were annotated using the Affymetrix annotation files from Netaffx (www.netaffx.com, HuEx-1_0-stv2.na29.hg18.probeset.csv).

The raw gene expression data were at first preprocessed by quartile normalization. Then the robust multi-array average values of every gene $(17,318$ 
measured genes) were adjusted for a set of technical covariates, for example, chip batch by fitting LME models. Imputed blood cell counts (that is white blood cell (WBC), red blood cell (RBC), platelet, lymphocyte, monocyte, eosinophil, and basophil) (Joehanes R, in preparation) were also evaluated as covariates and adjusted if deemed significant, as detailed below. The residuals were retained for further analysis.

Matching cis-miR-eQTLs with cis-mRNA-eQTLs. We overlapped the cis-miReQTLs at FDR $<0.1$ reported in this study with cis-mRNA-eQTLs at FDR $<0.1$ identifed by refs 9,25 . Hypergeometric test was used to evaluate if $c i s$-miR-eQTLs

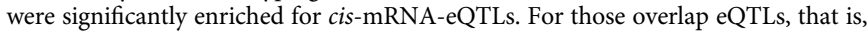
cis-miR-eQTLs that were also cis-mRNA-eQTLs, we used the same linear mixed regression model as described in 'miR-eQTL mapping' section to re-analyze the associations between genotypes and miRNA expression levels but conditional regression on corresponding mRNA expression levels.

Estimating effects of cell counts in the miR-eQTLs. Since the miR-eQTLs in whole blood may be driven by cellular composition, we compared the miR-eQTLs in 2,138 individuals with measured cell counts before and after correction for cell count effects (Supplementary Fig. 3). Differential cell counts and proportions in whole blood were measured in 2,138 individuals in the FHS third generation cohort, including seven cell types, WBC, RBC, platelet, neutrophil, lymphocyte, monocyte, eosinophil and basophil. The cell counts and proportions for 5,024 FHS participants were estimated using mRNA expression values by partial least squares regression prediction. The estimated cell count proportion values are highly consistent with the measured cell counts proportion values (Joehanes R, unpublished data, 2014).

We did not find any evidence that cell counts affected the miR-eQTLs; however, we cannot exclude small effects from cell counts. Therefore, we report miR-eQTLs unadjusted for cell counts in our main results, and secondarily report miR-eQTLs adjusted for imputed cell counts (that is, WBC, RBC, platelets, lymphocytes, monocytes, eosinophils and basophils) in Supplementary Data 5. Please note that there were 215 samples without mRNA expression data; therefore, the maximum sample size of analyses unadjusted for cell counts is 5,239 and the maximum sample size of analyses adjusted for cell counts is 5,024.

Estimating the heritability of miRNA expression levels. To estimate the narrow-sense heritability of the expression for a specific miRNA (denoted as $h_{\operatorname{miR}}^{2}$ ), we used the model as shown in Equation (3).

$$
\mathrm{miR}=\text { age }+\mathrm{sex}+\sum_{i=1}^{n} \text { tehnical covariates }+\mathrm{FAM}+\varepsilon
$$

Here age, sex and technical covariates were included as fixed effects, FAM was the familial correlation matrix included as the random effect. FAM-represented additive polygenic genetic effects ${ }^{39} . \varepsilon$ is the error term for each independent observation.

$h_{\mathrm{miR}}^{2}$ was the proportion of the additive polygenic genetic variance $\left(\sigma_{\mathrm{FAM}}^{2}\right)$ among the total phenotypic variance $\left(\sigma_{\mathrm{miR}}^{2}\right)$ of miRNA expression: $h_{\mathrm{miR}}^{2}=\sigma_{\mathrm{FAM}}^{2} / \sigma_{\mathrm{miR}}^{2}$. We estimated $h_{\mathrm{miR}}^{2}$ for every miRNA expression trait (247 miRNAs expressed in $>1,000$ samples) using the lmekin() function of Kinship package (http://cran.r-project.org/web/packages/kinship/) (39 $^{39}$.

Estimating proportion of variance in miRNAs attributable to miR-eQTLs. To estimate the proportion of variance in a single miRNA trait that is attributable to a single miR-eQTL (denoted as $h_{\text {miReQTL }}^{2}$ ), we used the following two models: Full model:

$$
\mathrm{miR}=\mathrm{SNP}+\text { age }+ \text { sex }+ \text { cohort }+\sum_{i=1}^{n} \text { techical covariates }+\mathrm{FAM}+\varepsilon
$$

Null model:

$$
\mathrm{miR}=\operatorname{age}+\mathrm{sex}+\text { cohort }+\sum_{i=1}^{n} \text { techical covariates }+\mathrm{FAM}+\varepsilon
$$

Here age, sex, cohort (offspring cohort and the third generation cohort in the FHS) and technical covariates were included as fixed effects, FAM was the familial correlation matrix included as the random effect. $\varepsilon$ is the error term for each independent observation. The proportion of variance in a single miRNA trait that is attributable to a single miR-eQTL was denoted as $h_{\text {miReQTL }}^{2}$ and was calculated as follows:

$$
h_{\text {miReQTL }}^{2}=\max \left(0, \frac{\sigma_{\text {QTL.null }}^{2}+\sigma_{\text {err.null }}^{2}-\sigma_{\mathrm{QTL} . \text { full }}^{2}-\sigma_{\text {err.full }}^{2}}{\sigma_{\mathrm{miR}}^{2}}\right)
$$

where $\sigma_{\mathrm{miR}}^{2}$ was the total phenotypic variance of a miRNA expression trait; $\sigma_{\mathrm{QTL} . f u l l}^{2}$ and $\sigma_{\text {err.full }}^{2}$ were the polygenic and error variances, respectively, when modelling with the tested miR-eQTL; $\sigma_{\mathrm{OTL} \text {.null }}^{2}$ and $\sigma_{\text {err.null }}^{2}$ were the polygenic and error variances, respectively, when modelling without the tested miR-eQTL. The $\operatorname{lmekin}()$ function in the Kinship package ${ }^{39}$ was used to estimate $h_{\text {miR-eQTL. }}^{2}$.
Identification of differentially expressed miRNAs for complex traits. We used the NHGRI GWAS Catalogue (http://www.genome.gov/gwastudies/) $)^{21}$ and NHLBI GRASP database (http://apps.nhlbi.nih.gov/grasp/22 to annotate complex trait associated miR-eQTLs. The cis-miR-eQTLs identified in this study were compared with SNPs in the NHGRI GWAS Catalogue and NHLBI GRASP GWAS results for SNPs at $P<1 \times 10^{-5}$.

For the complex traits that could be mapped with cis-miR-eQTLs (and also were measured in the FHS), including menarche, lipids (HDL cholesterol, TG and TC), type II diabetes mellitus (T2D) and glucose level, we used linear mixed models to test their association with miR-eQTL miRNAs in FHS individuals. These phenotypes were ascertained at examinations 8 and 2 for the offspring and the third generation cohorts, respectively. We identified differentially expressed miRNAs associated with HDL cholesterol, TC, TG, T2D and glucose after accounting for age, sex, cell counts and technical covariates (see 'miRNA normalization' in methods) and family structure in LME models implemented in the lmekin function ${ }^{39}$. Differentially expressed miRNA associated with age at menarche were tested in LME models (lmekin) after accounting for birth year, cell counts, technical covariates and family structure.

miRNA TSS and promoter regions. The transcriptional regulatory mechanisms affecting miRNA expression are unclear. There are technical barriers to the precise identification of primary miRNAs, TSSs and promoter regions for most mature miRNAs ${ }^{29}$. Recently, Marsico et al. ${ }^{29}$ and Chen et al. ${ }^{42}$ predicted miRNAs TSSs. Their results were incorporated with the results from previous similar studies ${ }^{43}$. However, by comparing the TSS positions identified by these two studies, there was, on average, $55 \mathrm{~kb}$ distance difference between TSSs positions to the corresponding mature miRNAs. Therefore, in our analysis, we annotated the miRNA TSSs collected and predicted by these two studies. The predicted promoter annotations for miRNAs were obtained from Marsico et al. ${ }^{29}$ which were screened within $\pm 50 \mathrm{~kb}$ from the TSSs for each miRNA.

Functional annotation of cis-miR-eQTLs. We annotated the genomic features cis-miR-eQTLs $(n=5,269)$ using HaploReg ${ }^{4}$, which integrates results from ENCODE $^{20}$. The overlap of $c i s$-miR-eQTLs with ENCODE annotated SNPs in promoter, enhancer and TF binding sites were retrieved (Supplementary Data 11).

For enrichment tests of functional SNPs in cis-miR-eQTLs identified in this study, we downloaded regulatory tracks contained in the UCSC Genome Browser, including ENCODE histone modification sites, and TF and CTCF binding sites in lymphoblastoid cell lines (GM12878), Open Regulatory Annotation (ORegAnno $)^{45}$, UCSC CpG islands and long intergenic non-coding RNA ${ }^{46}$. We also downloaded other regulatory tracks, including experimentally validated miRNA targets from TARbase ${ }^{47}$, and experimentally supported miRNA-mediated gene regulatory sites from Patrocles ${ }^{23}$. Binominal tests were used to evaluate if the identified cis-miR-eQTLs set (5,269 cis-miR-eQTLs) showed enrichment for regulatory SNPs for each track (methods described by ref. 13).

We further determined whether or not the detected cis-miR-eQTLs SNPs were enriched for promoter, enhancer or protein binding regions on the genome. To do so, we annotated all cis-miR-eQTLs $(n=5,269)$ using HaploReg ${ }^{44}$, which integrates results from $\mathrm{ENCODE}^{20}$. We examined enrichment in nine different cell lines (that is, GM12878, H1-hESC, HepG2, HMEC, HSMM, HUVEC, K562, NHEK and NHLF). The null distributions of eQTLs were generated using a permutation strategy by randomly selecting equal number of SNPs $(n=5,269) 100$ times. The pools of candidate SNPs for the permutation were from 1000-genomes imputed SNPs with MAF $>0.01$ and imputation quality ratio $>0.1$. To match the distribution of MAFs of the permutation SNPs (the permutation-SNPs set) with the cis-miR-eQTLs SNPs (the tested-SNPs set), we categorized MAF into four categories: MAF of $(0.01,0.05),(0.05,0.1),(0.1,0.2)$ and $(0.2,0.5)$. For each MAF category, we kept the proportion of SNPs in the permutation-SNPs set equal to the proportion of SNPs in the tested-SNPs set. In the four MAF categories, the proportions of SNPs are $3 \%, 7 \%, 19 \%$ and $71 \%$ respectively. The average of the overlap between permutation and regulatory region SNPs (that is SNPs in promoter, enhancer and protein binding regions) was compared with the overlap between the tested SNPs and regulatory region SNPs.

MR test. We used a two-stage least squares (2SLS) MR method ${ }^{48}$ to estimate the causal relationships between miRNAs and complex traits measured in FHS participants; the traits analyzed included menarche, lipids (HDL, TG and TC), T2D and glucose, using cis-miR-eQTLs as instrumental variables. MR was only performed in the pre-filtered SNP-miRNA-trait pairs, when a SNP was a cis-miR-eQTL and also present in NHGRI GWAS Catalogue (http://www.genome.gov/gwastudies/) $)^{21}$ or in the NHLBI GRASP database (http://apps.nhlbi.nih.gov/grasp/) ${ }^{22}$, and the miRNA showed differential expression in relation to the corresponding trait at $P<0.05$ in FHS participants.

To determine the strength of the genetic instrument, an F-statistic in a linear regression model was derived from the proportion of variation in the miRNA expression levels (miRNA Ct values) that was explained by the corresponding cis-miR-eQTL, by modelling age, sex, family structure and four technical variables as covariates (see in the miRNA normalization section). cis-miR-eQTLs with an F-statistic $<10$, indicating a weak instrument, were excluded. The first stage of the 
2SLS method involves using a linear regression of the modifiable exposure (miRNA) on the instrumental variable (SNP) and covariates, and saving the predicted miRNA values. In the second stage, the outcome (complex trait) is regressed on the predicted miRNA values. The regression coefficient obtained in the second stage can be interpreted as being the causal effect of the exposure (miRNA) on the outcome (complex trait). The Durbin-Wu-Hausman test ${ }^{49}$ is used to estimate whether the estimates derived from the first and second stage of the 2SLS are consistent.

\section{References}

1. Lee, R., Feinbaum, R. \& Ambros, V. A short history of a short RNA. Cell 116, S89-S92 (2004)

2. Lee, R. C. \& Ambros, V. An extensive class of small RNAs in Caenorhabditis elegans. Science 294, 862-864 (2001).

3. Cordes, K. R. \& Srivastava, D. MicroRNA regulation of cardiovascular development. Circ. Res. 104, 724-732 (2009).

4. Small, E. M. \& Olson, E. N. Pervasive roles of microRNAs in cardiovascular biology. Nature 469, 336-342 (2011).

5. Thum, T. et al. MicroRNAs in the human heart: a clue to fetal gene reprogramming in heart failure. Circulation 116, 258-267 (2007).

6. Tijsen, A. J. et al. MiR423-5p as a circulating biomarker for heart failure. Circ. Res. 106, 1035-1039 (2010).

7. Fiedler, J. \& Thum, T. MicroRNAs in myocardial infarction. Arterioscler Thromb. Vasc. Biol. 33, 201-205 (2013).

8. Lu, J. et al. MicroRNA expression profiles classify human cancers. Nature 435, 834-838 (2005)

9. Westra, H.-J. et al. Systematic identification of trans eQTLs as putative drivers of known disease associations. Nat. Genet. 45, 1238-1243 (2013).

10. Zhang, B. et al. Integrated systems approach identifies genetic nodes and networks in late-onset Alzheimer's disease. Cell 153, 707-720 (2013).

11. Emilsson, V. et al. Genetics of gene expression and its effect on disease. Nature 452, 423-428 (2008).

12. Schadt, E. E. et al. Mapping the genetic architecture of gene expression in human liver. PLoS Biol. 6, e107 (2008).

13. Zhang, X. et al. Synthesis of 53 tissue and cell line expression QTL datasets reveals master eQTLs. BMC Genomics 15, 532 (2014).

14. Borel, C. et al. Identification of cis- and trans-regulatory variation modulating microRNA expression levels in human fibroblasts. Genome Res. 21, 68-73 (2011).

15. Gamazon, E. R. et al. Genetic architecture of microRNA expression: implications for the transcriptome and complex traits. Am. J. Hum. Genet. 90, 1046-1063 (2012).

16. Civelek, M. et al. Genetic regulation of human adipose microRNA expression and its consequences for metabolic traits. Hum. Mol. Genet. 22, 3023-3037 (2013).

17. Somel, M. et al. MicroRNA, mRNA, and protein expression link development and aging in human and macaque brain. Genome Res. 20, 1207-1218 (2010).

18. Siddle, K. J. et al. A genomic portrait of the genetic architecture and regulatory impact of microRNA expression in response to infection. Genome Res. 24, 850-859 (2014)

19. Abecasis, G. R. et al. An integrated map of genetic variation from 1,092 human genomes. Nature 491, 56-65 (2012).

20. Birney, E. et al. Identification and analysis of functional elements in $1 \%$ of the human genome by the ENCODE pilot project. Nature 447, 799-816 (2007).

21. Hindorff, L. A. et al. Potential etiologic and functional implications of genomewide association loci for human diseases and traits. Proc. Natl Acad. Sci. USA 106, 9362-9367 (2009).

22. Leslie, R., O’Donnell, C. J. \& Johnson, A. D. GRASP: analysis of genotypephenotype results from 1390 genome-wide association studies and corresponding open access database. Bioinformatics 30, i185-i194 (2014).

23. Hiard, S., Charlier, C., Coppieters, W., Georges, M. \& Baurain, D. Patrocles: a database of polymorphic miRNA-mediated gene regulation in vertebrates. Nucleic Acids Res. 38, D640-D651 (2010).

24. Hsu, S. D. et al. miRTarBase: a database curates experimentally validated microRNA-target interactions. Nucleic Acids Res. 39, D163-D169 (2011).

25. Joehanes, R. et al. Genome-wide Expression Quantitative Trait Loci: Results from the NHLBI s SABRe CVD Initiative. in the American Society of Human Genetics (ASHG) Conference. (Boston, MA, USA, 2013).

26. Huan, T. et al. A systematic heritability analysis of the human whole blood transcriptome. Human genetics 134, 343-358 (2015).

27. Cesana, M. et al. A long noncoding RNA controls muscle differentiation by functioning as a competing endogenous RNA. Cell 147, 358-369 (2011).

28. Degner, J. F. et al. DNase I sensitivity QTLs are a major determinant of human expression variation. Nature 482, 390-394 (2012).

29. Marsico, A. et al. PROmiRNA: a new miRNA promoter recognition method uncovers the complex regulation of intronic miRNAs. Genome Biol. 14, R84 (2013).

30. Chen, B. H. et al. Transcriptome-wide association study of circulating lipid levels. Circulation 129, A35-A35 (2014).

31. Feinleib, M., Kannel, W. B., Garrison, R. J., McNamara, P. M. \& Castelli, W. P. The Framingham Offspring Study. Design and preliminary data. Prev. Med. 4, 518-525 (1975)
32. Splansky, G. L. et al. The third generation cohort of the national heart, lung, and blood institute's Framingham heart study: design, recruitment, and initial examination. Am. J. Epidemiol. 165, 1328-1335 (2007).

33. Chen, C., Tan, R., Wong, L., Fekete, R. \& Halsey, J. Quantitation of microRNAs by real-time RT-qPCR. Methods Mol. Biol. 687, 113-134 (2011).

34. Chen, C. et al. Real-time quantification of microRNAs by stem-loop RT-PCR. Nucleic Acids Res. 33, e179 (2005).

35. Jensen, S. G. et al. Evaluation of two commercial global miRNA expression profiling platforms for detection of less abundant miRNAs. BMC Genomics 12, 435 (2011).

36. Jang, J. et al. Quantitative miRNA expression analysis using fluidigm microfluidics dynamic arrays. BMC Genomics 12, 144 (2011).

37. Levy, D. et al. Genome-wide association study of blood pressure and hypertension. Nat. Genet. 41, 677-687 (2009).

38. Trapnell, C., Pachter, L. \& Salzberg, S. L. TopHat: discovering splice junctions with RNA-Seq. Bioinformatics 25, 1105-1111 (2009).

39. Almasy, L. \& Blangero, J. Multipoint quantitative-trait linkage analysis in general pedigrees. Am. J. Hum. Genet. 62, 1198-1211 (1998).

40. Benjamini, Y. \& Hochberg, Y. Controlling the false discovery rate: a practical and powerful approach to multiple testing. J. R. Stat. Soc. Ser. B 57, 289-300 (1995).

41. Qin, Z. S., Gopalakrishnan, S. \& Abecasis, G. R. An efficient comprehensive search algorithm for tagSNP selection using linkage disequilibrium criteria. Bioinformatics 22, 220-225 (2006).

42. Chen, D. et al. Dissecting the chromatin interactome of microRNA genes. Nucleic Acids Res. 42, 3028-3043 (2014).

43. Chien, C. H. et al. Identifying transcriptional start sites of human microRNAs based on high-throughput sequencing data. Nucleic Acids Res. 39, 9345-9356 (2011).

44. Ward, L. D. \& Kellis, M. HaploReg: a resource for exploring chromatin states, conservation, and regulatory motif alterations within sets of genetically linked variants. Nucleic Acids Res. 40, D930-D934 (2012).

45. Griffith, O. L. et al. ORegAnno: an open-access community-driven resource for regulatory annotation. Nucleic Acids Res. 36, D107-D113 (2008).

46. Cabili, M. N. et al. Integrative annotation of human large intergenic noncoding RNAs reveals global properties and specific subclasses. Genes Dev. 25, 1915-1927 (2011).

47. Vergoulis, T. et al. TarBase 6.0: capturing the exponential growth of miRNA targets with experimental support. Nucleic Acids Res. 40, D222-D229 (2012).

48. Lawlor, D. A., Harbord, R. M., Sterne, J. A., Timpson, N. \& Davey Smith, G. Mendelian randomization: using genes as instruments for making causal inferences in epidemiology. Stat. Med. 27, 1133-1163 (2008).

49. Baum, C. F., Schaffer, M. E. \& Stillman, S. IVENDOG: Stata module to calculate Durbin-Wu-Hausman endogeneity test after ivreg. Statistical Software Components, Department of Economics, Boston College (2007).

50. Teslovich, T. M. et al. Biological, clinical and population relevance of 95 loci for blood lipids. Nature 466, 707-713 (2010).

\section{Acknowledgements}

The Framingham Heart Study is funded by the National Institutes of Health contract N01-HC-25195. The laboratory work for this investigation was funded by the Division of Intramural Research, National Heart, Lung, and Blood Institute, the National Institutes of Health. The analytical component of this project was funded by the Division of Intramural Research, the National Heart, Lung, and Blood Institute, and the Center for Information Technology, the National Institutes of Health, Bethesda, MD, USA. This study utilized the high-performance computational capabilities of the Biowulf Linux cluster at the National Institutes of Health, Bethesda, MD, USA (http://biowulf.nih.gov).

\section{Author contributions}

D.L. and J.E.F. designed, directed and supervised the project. D.L., M.G.L and J.E.F. were responsible for funding of the project. T.H and D.L. drafted the manuscript. J.E.F. and K.T. directed and supervised the miRNA experiment. P.C. organized the experiment material and data exchange. All authors participated in revising and editing the manuscripts. All authors have read and approved the final version of the manuscript.

\section{Additional information}

Accession codes: The mRNA and microRNA expression data of patient samples have been deposited in $\mathrm{dbGaP}$ (http://www.ncbi.nlm.nih.gov/gap) under the accession number phs000007.

Supplementary Information accompanies this paper at http://www.nature.com/ naturecommunications

Competing financial interests: There are no competing financial interests.

Reprints and permission information is available online at http://npg.nature.com/ reprintsandpermissions/

How to cite this article: Huan, T. et al. Genome-wide identification of microRNA expression quantitative trait loci. Nat. Commun. 6:6601 doi: 10.1038/ncomms7601 (2015). 